\title{
Exploration of the profile-effect relationship of Siraitia grosvenorii aqueous extracts related to their laxative effect on the basis of gray correlation analysis
}

Wei Dong ${ }^{1+}$, Jia Zeng ${ }^{2+}$, Qin Wang ${ }^{3}$, Xin Jiang $^{1 *}$ and Ting Huang ${ }^{2^{*}}$ (1)

\begin{abstract}
Background: Siraitia grosvenorii (binomial name Siraitia grosvenorii (Swingle) C. Jeffrey ex Lu et Z. Y. Zhang), also called Arhat Fruit or Monk's Fruit, is a dried ripe fruit belonging to the Cucurbitaceae Family. S. grosvenorii has a long history of being used for constipation treatment in folk medicine. However, there are few studies where the laxative effect, related mechanisms, and active constituents of S. grosvenorii were investigated. This research explores the relationship between the common components and the laxative effect of aqueous extracts of S. grosvenorii from different habitats in China.
\end{abstract}

Methods: The fingerprints of S. grosvenorii aqueous extracts from different habitats were established by HPLC. The constipation mice model was used to investigate the laxative effect of S. grosvenorii aqueous extracts. The motilin (MTL) level in mice serum, and the water content of the large and small intestines in mice were determined. The profile-effect relationship of $S$. grosvenorii aqueous extracts was preliminarily clarified using gray correlation analysis.

Results: Nine common peaks were identified from the fingerprint of aqueous extracts of S. grosvenorii. The aqueous extracts obviously shortened the incubation period of defecation, and significantly increased the number of defecations, and the wet and dry weight of defecation in constipated mice. The profile-effect relationship indicated that seven common peaks were highly correlated with the effect of the incubation period of defecation, the number of defecations, and the wet and dry weight of defecation in mice.

Conclusion: This work provides a promising method for the fingerprint establishment, pharmacodynamic evaluation, and quality control of S. grosvenorii on the basis of its profile-effect relationship.

Keywords: Siraitia grosvenorii, Fingerprint, Laxative effect, Profile-effect relationship, Gray correlation analysis

\footnotetext{
*Correspondence: 1024109626@qq.com; ting-lemon@163.com

${ }^{+}$Wei Dong and Jia Zeng contributed equally to this work.

'Department of Pharmacy, The Affiliated Hospital of Jiaxing University,

Department of Pharmacy, The First Hospital of Jiaxing, Jiaxing 314000,

Zhejiang Province, PR China

${ }^{2} \mathrm{NHC}$ Key Laboratory of Reproduction Regulation, Shanghai Engineering

Research Center of Reproductive Health Drug and Devices, Shanghai Institute of Planned Parenthood Research, Shanghai 200032, PR China

Full list of author information is available at the end of the article
}

(c) The Author(s). 2021 Open Access This article is licensed under a Creative Commons Attribution 4.0 International License, which permits use, sharing, adaptation, distribution and reproduction in any medium or format, as long as you give appropriate credit to the original author(s) and the source, provide a link to the Creative Commons licence, and indicate if changes were made. The images or other third party material in this article are included in the article's Creative Commons licence, unless indicated otherwise in a credit line to the material. If material is not included in the article's Creative Commons licence and your intended use is not permitted by statutory regulation or exceeds the permitted use, you will need to obtain permission directly from the copyright holder. To view a copy of this licence, visit http://creativecommons.org/licenses/by/4.0/ The Creative Commons Public Domain Dedication waiver (http://creativecommons.org/publicdomain/zero/1.0/) applies to the data made available in this article, unless otherwise stated in a credit line to the data. 
Constipation can lead to the continuous absorption of toxic substances, stimulate the local mucosa, and may cause malignant disease potentially in intestine. Natural products have long been and still are the source of treatments of different digestive disorders including cancer [1-5]. S. grosvenorii has a long history of being used for constipation treatment in folk medicine. In the present study, 624 ICR mice were approved to carry out the experiment by the Animal Research Ethics approval, and the animals were killed by carbon dioxide asphyxiation. This research explores the relationship between the common components and the laxative effect of aqueous extracts of S. grosvenorii from different habitats in China. S. grosvenorii from some places of origin were observed to significantly shorten the incubation period of defecation, and significantly increase the number of defecations, and the wet and dry weights of defecation in constipated mice. A potential explanation for the laxative effect of the aqueous extracts of S. grosvenorii was related to their ability to increase the MTL level in the serum of mice, and thus promote gastric emptying and intestinal propulsion. Further, it increased the water content of the small intestine, which supported its effect on bowel relaxation.

\section{Background}

Siraitia grosvenorii (binomial name Siraitia grosvenorii (Swingle) C. Jeffrey ex Lu et Z. Y. Zhang), also called Arhat Fruit or Monk's Fruit, is a dried ripe fruit belonging to the Cucurbitaceae Family [6]. As a famous edible and medicinal plant, S. grosvenorii has been cultivated for more than 200 years in China. It is abundant in many areas, such as northern Guangxi (mostly in the mountains of Guilin), and especially in Yongfu and Lingui counties (called "genuine medicinal materials"), as well as in Guangdong, Guizhou, Hunan, and Jiangxi [7]. Furthermore, it is a traditional export commodity of China and has been sold to more than 20 countries, such as America, Japan and several in south-east Asia [7]. In folk medicine applications, it shows definite curative effects in treating pertussis, chronic bronchitis, pharyngitis, and constipation.

Over the last 40 years, S. grosvenorii has been proven to contain many chemical constituents, including triterpene glycosides [8, 9], flavonoids [10, 11], proteins, vitamins, sugars, inorganic elements, and volatile oils [8]. Currently, cucurbitane triterpenoids, such as mogroside $\mathrm{V}$, are considered to be the main active components of $S$. grosvenorii. Furthermore, modern pharmacological and clinical investigations have demonstrated that $S$. grosvenorii acts as an antitussive and expectorant [12$14]$, it stimulates the immune system [15, 16], offers liver protection [17, 18], eliminates free radicals [19], regulates blood sugar and blood fat levels [20-22], and has anti-inflammatory [23], anti-carcinogenic [24], and antifatigue effects [25]. In general, previous studies on $S$. grosvenorii mainly focused on the extraction and isolation of its chemical components and the screening and evaluation of its pharmacological activities. As previously described, S. grosvenorii has a long history of being used for constipation treatment in folk medicine. However, there are few studies where the laxative effect, related mechanisms, and active constituents of S. grosvenorii were investigated.

The present study is the first to clarify the profileeffect relationship of S. grosvenorii aqueous extracts related to their laxative effect. The relationship of the fingerprint of $S$. grosvenorii aqueous extracts with their laxative effect not only bridges the gap between the fingerprint and the observed pharmacodynamic effects, but also helps to establish the correlation between them. This study provides a promising method for the establishment of a fingerprint database, pharmacodynamic evaluation, and quality control of $S$. grosvenorii on the basis of its profile-effect relationship.

\section{Methods}

\section{Chemicals and reagents}

Mogroside V with a purity of $98 \%$ was purchased from Nanjing Spring \& Autumn Biological Engineering Co., Ltd. (Nanjing, China). Chromatographic-grade acetonitrile and phosphoric acid were purchased from SigmaAldrich (St. Louis, Mo.). Diphenoxylate tablets were purchased from Changzhou Green Peak Environmental Protection Tech (50 mg/tablet, Changzhou, China). Mosapride citrate dispersible tablets were purchased from Chengdu Kanghong Pharmaceutical Group Co., Ltd. (5 mg/tablet, Chengdu, China). Activated charcoal was supplied by Chengdu Changzheng Glass Co., Ltd. (Chengdu, China). The mouse motilin (MTL) ELISA kit was supplied by Wuhan Boster Biological Technology Co., Ltd. (Wuhan, China). The other reagents were of analytical grade and commercially available.

\section{Animals}

ICR mice with a body weight of $18-22 \mathrm{~g}$ were obtained from Hunan Slake Jingda Experimental Animal Co., Ltd. (Hunan, China). The animals were fed in a standard feeding room maintained from 18 to $26^{\circ} \mathrm{C}$ at a relative humidity of $50-70 \%$. The animals were killed by carbon dioxide asphyxiation after the experiments. All experiments were performed according to the guidelines for the care and use of animals established by Guangxi University of Chinese Medicine. All animal studies were in line with the guidelines of the Animal Ethics Committee of Guangxi University of Chinese Medicine. 
(a)

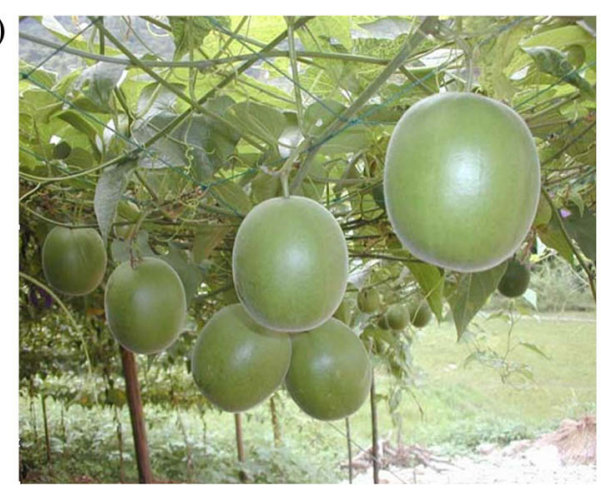

(b)

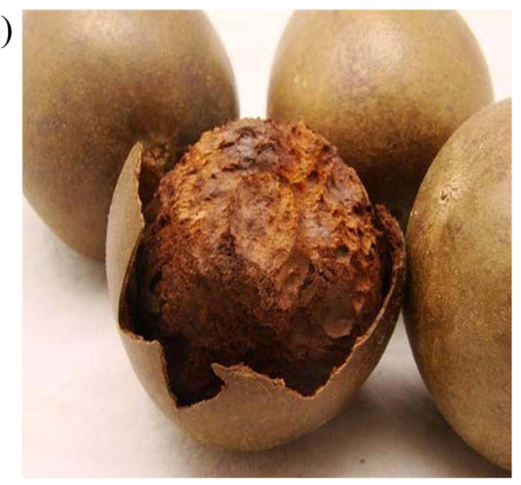

Fig. 1 Appearance of S. Grosvenorii (a) fresh fruits; (b) dried fruits

\section{Collection and identification of S. grosvenorii}

A total of 25 batches of S. grosvenorii from different ecological regions or from the same region with different growth phases, breeding type, and sizes were collected in Guangxi and Hunan Provinces, China. The samples were identified as the fruit of Momordica grosvenorii Swingle by Professor Tian Hui from Guangxi University of Chinese Medicine. The appearance of $S$. grosvenorii is shown in Fig. 1 and the detailed sample information is summarized in Table 1 (Supplementray file).

\section{Preparation of S. grosvenorii aqueous extracts}

Water extraction and an alcohol precipitation method were used to prepare $S$. grosvenorii aqueous extracts as follows. First, the herbs were weighed and broken into small pieces. Then, they were decocted three times with 10,8 , and 5 fold water for $1.5 \mathrm{~h}, 1 \mathrm{~h}$, and $1 \mathrm{~h}$, respectively. Before the first extraction, the herbs were soaked for $0.5 \mathrm{~h}$ in water. After decocting, the extracted solutions were blended, filtered, and concentrated to a relative density of $1.05 \mathrm{~g} / \mathrm{mL}$. Then an equal volume of $95 \%$ ethanol was added and the mixture was refrigerated for $48 \mathrm{~h}$ undisturbed to allow the solid components to deposit at the bottom of the container. Finally, the ethanol-deposition components were filtered and concentrated to a paste for analysis.

\section{HPLC method for fingerprinting the S. grosvenorii aqueous extracts}

The fingerprint of the S. grosvenorii aqueous extracts was established using an Agilent 1100 series HPLC system (Agilent Technologies, Santa Clara, CA, USA). Mogroside V and S. grosvenorii aqueous extracts were dissolved in methanol to prepare the reference solution and the test solution, respectively. A ZORBAX SB-C18 column $(4.6 \times 150 \mathrm{~mm}, 5 \mu \mathrm{m})$ maintained at a flow rate of $0.8 \mathrm{~mL} / \mathrm{min}$ and $20{ }^{\circ} \mathrm{C}$ was used for the separation. The mobile phase consisted of $0.05 \%$ phosphoric acid (A) and acetonitrile (B) (0-8 min, 3.0-13.5\% B; 8.0-35.0 min, $13.5-35.0 \% \mathrm{~B}$; $35.0-45.0 \mathrm{~min}, 35.0 \% \mathrm{~B})$. A $10 \mu \mathrm{L}$ aliquot of the sample was injected into the system and the components detected at $203 \mathrm{~nm}$. The total run time was $45 \mathrm{~min}$.

\section{Investigation of the laxative effect of S. grosvenorii aqueous extracts}

A mouse constipation model induced by diphenoxylate was used to investigate the laxative effect of S. grosvenorii aqueous extracts from different areas of Guangxi and Hunan Provinces, China. Male ICR mice were randomly divided into 28 groups, 18 mice in each group. The model groups were orally administered a diphenoxylate solution at a dose of $10 \mathrm{mg} / \mathrm{kg}$ and the negative control group was orally administered an equal volume of pure water. Before the experiment, the animals were fasted for $12 \mathrm{~h}$ with access to drinking water. Except for the negative control group, the other mice were given $10 \mathrm{mg} / \mathrm{kg}$ diphenoxylate solution by gavage $(0.2 \mathrm{~mL} / 10 \mathrm{~g}$, volume/body weight) once a day for 3 consecutive days to construct the constipation model. A total of $40 \mathrm{~min}$ after the diphenoxylate dose, the model mice were treated with an equal volume of pure water, $10 \mathrm{mg} / \mathrm{kg}$ mosapride solution, and $30 \mathrm{~g} / \mathrm{kg} \mathrm{S}$. grosvenorii aqueous extracts from different areas. After another $40 \mathrm{~min}$, the mice were orally administered a 5\% activated charcoal solution. The incubation period of defecation, the number of defecations, the wet weight of defecation, and the dry weight of defecation over the following $3 \mathrm{~h}$ were then recorded. The animals were killed by carbon dioxide asphyxiation when the experiments were finished.

\section{Determination of the MTL level in the serum of normal mice}

A total of 80 male mice weighing $18-22 \mathrm{~g}$ were randomly divided into 5 groups with 16 per group. The groups included the blank control group, the positive control group, and the $S$. grosvenorii aqueous extract group treated with low, middle, and high doses $(15 \mathrm{~g} / \mathrm{kg}$, 
$30 \mathrm{~g} / \mathrm{kg}$, and $45 \mathrm{~g} / \mathrm{kg}$, respectively, administered once a day for 10 consecutive days). At $1 \mathrm{~h}$ after the last administration, $1 \mathrm{~mL}$ of blood was collected from the eyeball and placed in a centrifugation tube. The animals were killed by carbon dioxide asphyxiation. The blood coagulated naturally at room temperature for 10-20 min, and then was centrifuged $(3000 \mathrm{rpm})$ at $4{ }^{\circ} \mathrm{C}$ for $20 \mathrm{~min}$. The supernatant was then separated and the mouse serum was prepared.

A MTL ELISA kit was used to determine the MTL level in the mouse blood serum. The level of MTL was determined by a double-antibody sandwich method. The purified mouse MTL antibody was coated on a microporous plate to create a solid-phase antibody. MTL was added to the coated plate, and a horseradish peroxidase (HRP)-labeled MTL antibody was added to form the antibody, antigen, and enzyme-labeled antibody complex. After thorough washing, the substrate tetramethylbenzidine (TMB) was added to determine the amount of MTL antigen captured. TMB was first converted to blue by HRP, and then to yellow in the presence of acid. There was a positive correlation between the color and MTL level in the samples. The optical density (OD) value was measured at $450 \mathrm{~nm}$ using a microplate reader within $15 \mathrm{~min}$ after adding the termination solution. The MTL level in the serum of mice was calculated by a standard curve. The operation steps were in strict accordance with the instructions of the MTL ELISA Kit.

\section{Determination of the water content in the small and large intestines of normal mice}

The effect of $S$. grosvenorii aqueous extracts on the water content of the large and small intestines in normal mice were tested by a weighing method. A total of 40 mice, half male and half female, were randomly divided into the blank control group and the S. grosvenorii aqueous extracts group with 20 in each group. After $18 \mathrm{~h}$ of fasting, one group was treated with $S$. grosvenorii aqueous extracts at a dose of $45 \mathrm{~g} / \mathrm{kg}$ and the blank control group was administered an equal volume of distilled water. After $75 \mathrm{~min}$, the mice were put to death by carbon dioxide asphyxiation. The abdominal cavity was opened and the pylorus, ileocecal, and rectum were ligated. Next, the ligated pylorus, ileocecal and rectum were cut to ensure that the water in the intestinal cavity did not flow out. The wet weights of the large and small intestines of each mouse were determined and the intestines were stored on a square tray. The dry weight was weighed after heating at $80^{\circ} \mathrm{C}$ for $6 \mathrm{~h}$. The water content in the small and large intestines of mice was calculated according to Formula (1).
Water content $(\mathrm{g})=$ Wet weight $(\mathrm{g})$-Dry weight $(\mathrm{g})$

\section{Profile-effect relationship investigation}

The profile-effect relationship of S. grosvenorii aqueous extracts related to their laxative effect was investigated using a gray correlation analysis. The detailed steps were as follows.

\section{Step 1: selection of the reference and comparison sequences}

According to gray system theory, the chemical compositions of $S$. grosvenorii aqueous extracts were regarded as a whole. Different common peaks were regarded as the subsystems, and the relative peak area of each characteristic component was calculated as a gray element. The laxative effect of $S$. grosvenorii aqueous extracts including the incubation period of defecation, the number of defecations, the wet weight of defecation, and the dry weight of defecation were treated as the reference sequence denoted by $\mathrm{X} 0$ as shown in Formula (2). The main characteristic components of $S$. grosvenorii aqueous extracts were treated as the comparison sequence denoted by Xi as shown in Formula (3).

$$
\mathrm{X} 0=[\mathrm{X} 0(\mathrm{l}), \mathrm{X} 0(2), \mathrm{X} 0(3), \ldots \mathrm{X} 0(\mathrm{k})]
$$

Where k represents Siraitia grosvenorii from different regions, $\mathrm{k}=25$.

$$
\mathrm{Xi}(\mathrm{k})=[\mathrm{Xi}(1), \mathrm{Xi}(2), \mathrm{Xi}(3), \ldots \mathrm{Xi}(25)]
$$

\section{Step 2: calculation of the gray correlation coefficient}

According to the determined reference and comparison sequences, the calculation formula of the gray correlation coefficient is shown in Formula (4).

$$
\varepsilon_{i}(k)=\frac{\min _{i} \min _{k}|X 0(k)-X i(k)|+\rho \cdot \max _{i} \max _{k}|X 0(k)-X i(k)|}{|X 0(k)-X i(k)|+\rho \cdot \max _{i} \max _{k}|X 0(k)-X i(k)|}
$$

Where k represents $S$. grosvenorii from different regions, $\mathrm{X}_{\mathrm{i}}$ represents the main characteristic components of $S$. grosvenorii aqueous extracts, $\varepsilon_{i}$ represents the correlation coefficient between the comparison and the reference sequences of the k-th batch of $S$. grosvenorii. $\min _{i} \min _{k}|X 0(k)-X i(k)|$ represents the minimum difference between the comparison and the reference sequences, denoted by $\Delta \mathrm{min}$.

$\max _{i} \max _{k}|X 0(k)-X i(k)|$ represents the maximum difference between the comparison and the reference 
sequences, denoted by $\Delta_{\max }|X 0(k)-X i(k)|$ represents the absolute difference between the comparison and the reference sequences, denoted by $\Delta \mathrm{i}(\mathrm{k}) . \rho$ is the resolution coefficient, which reduces the distortion in calculation caused by $\max _{i} \max _{k}|X 0(k)-X i(k)|$ and thus improves the significance of the difference between the correlation coefficients. The value range of $\rho$ is usually between 0 and 1 . In this study, it was set as 0.5 .

\section{Step 3: Calculation of the gray correlation grade}

The gray correlation grade is the arithmetic mean of the gray correlation coefficient shown in Formula (5).

$$
\mathrm{ri}=\frac{1}{N} \sum_{k=1}^{n} \varepsilon i(k)
$$

The relevancy grade of the reference sequence and each comparison sequence were rearranged into a row according to the relevancy order, which reflects the correlation grade of each comparison sequence to the reference sequence. The higher the correlation grade is, the closer the relationship is between the two characteristic components of $S$. grosvenorii aqueous extracts. As previously described, the laxative effect of $S$. grosvenorii aqueous extracts were treated as the reference sequence, and the nine common peaks (P1, P2, P3, P4, P5, P6, P7, P8, and $\mathrm{P} 9$ ) were treated as the comparison sequence. They were analyzed by the gray correlation method.

\section{Step 4: dimensionless treatment}

Since each sequence has different units or dimensions, each sequence should be dimensionless before performing the correlation analysis so as to achieve dimensional consistency. In the present study, a mean transformation was used to form new data columns. Namely, the average value of each sequence data was calculated, and then it was divided by the raw data in the corresponding sequence.

\section{Step 5: calculation of the absolute difference}

The absolute difference $\Delta \mathrm{i}(\mathrm{k})$ between the reference sequence and the comparison sequence was calculated according to the dimensionless value as shown in Formula (6).

$$
\Delta \mathbf{i}(\mathbf{k})=|X 0(k)-X i(k)| .
$$

\section{Step 6: calculation of the correlation coefficients between the characteristic peaks and the laxative effect}

According to the calculation results from step 5, the value of $\Delta \max$ and $\Delta \min (\Delta \max =8.515745, \Delta \min =0)$ were substituted into Formula (4). Then the correlation coefficients between the characteristic peaks and the laxative effect were obtained.

\section{Data analysis}

In the gray correlation analysis, data were processed and analyzed using the data analysis function of Microsoft Excel.

\section{Results}

HPLC fingerprint of $S$. grosvenorii aqueous extracts from different batches

Using the established chromatographic conditions, the HPLC fingerprint of 25 batches of $S$. grosvenorii aqueous extract and the common peaks are shown in Fig. 2. In the $S$. grosvenorii aqueous extracts from different regions, nine common fingerprint peaks were identified and named Peak 1 (P1), Peak 2 (P2), Peak 3 (P3), Peak 4 (P4), Peak 5 (P5), Peak 6 (P6), Peak 7 (P7), Peak 8 (P8), and Peak 9 (P9) according to the order of their retention times. Among them, P7 was determined to be mogroside $\mathrm{V}$ based on a comparison of the retention time and ultraviolet spectrum of a reference sample of mogroside $\mathrm{V}$. The identities of the other eight common peaks remain unknown.

The laxative effect of $S$. grosvenorii aqueous extracts from different batches

The effects of S. grosvenorii aqueous extracts from different batches on the constipation model of mice induced by diphenoxylate are shown in Figs. 3, 4, 5 and 6. Compared with the blank control group, the incubation period of defecation in the model group was significantly prolonged $(P<0.01)$, while the number of defecations, the wet weight of defecation, and the dry weight of defecation were significantly reduced $(P<0.01)$, indicating that the constipation model was successfully constructed. S. grosvenorii aqueous extracts from different batches exhibited different therapeutic effects on mice with constipation. Figure 3 shows that S1-S4, S6-S17, S19-S20, S22-S23, and S25 obviously shortened the incubation period of defecation in constipated mice $(P<$ 0.01 ), and were equivalent or more effective than the positive control. Figure 4 shows that S1-S9, S11, S13S17, S20, S22-S23, and S25 significantly increased the number of defecations in constipated mice $(P<0.01)$. Figure 5 shows that S4, S11, S14-S17, S20, and S23 significantly increased the wet weight of defecation in constipated mice $(P<0.01)$, of which S4, S11, S14-17, and S20 were equivalent or more effective than the positive control. Figure 6 shows that S1-S2, S4-S6, S11-S17, S20, and S23 significantly increased the dry weight of defecation in constipated mice $(P<0.01)$, of which S14S16, S20, and S23 were equivalent or more effective than the positive control. In general, S4, S11, S14-S17, S20, 
and S23 all obviously shortened the incubation period of defecation $(\mathrm{P}<0.01)$, and significantly increased the number of defecations, the wet weight of defecation, and the dry weight of defecation in constipated mice $(\mathrm{P}<$ 0.01). The large differences in the laxative effect of $S$. grosvenorii aqueous extracts from different batches may be attributed to the different metabolic fingerprints, and the latter were affected by different ecological environments or different growth phases, breeding type and/or sizes.

\section{Effect of S. grosvenorii aqueous extracts on the MTL level in serum of normal mice}

The effect of $S$. grosvenorii aqueous extracts on the MTL level in the serum of normal mice is shown in Fig. 7. Mosapride at a dose of $10 \mathrm{mg} / \mathrm{kg}$ and $S$. grosvenorii aqueous extracts, whether administered at a low, moderate or high dose, significantly increased the content of MTL in the serum of normal mice $(P<0.01)$. The effect of $S$. grosvenorii aqueous extracts on the MTL level in the serum of normal mice was dose-dependent and the effect of the high dose group was similar to that of mosapride. The present results indicated that S. grosvenorii aqueous extracts promoted gastric emptying and intestinal peristalsis.

\section{Effect of S. grosvenorii aqueous extracts on water} absorption of large and small intestines in normal mice The effect of $S$. grosvenorii aqueous extracts on the water absorption of the large and small intestines in normal mice is shown in Fig. 8. Compared with the blank control group, the high dose of $S$. grosvenorii aqueous extracts increased the water content of the large intestine in normal mice, but the difference was not significant $(P>0.05)$. By comparison, the same dose of $S$. grosvenorii aqueous extracts increased the water content of the small intestine in normal mice with a significant difference $(P<0.05)$. These results suggested that one of the primary mechanisms of $S$. grosvenorii aqueous extracts related to their laxative effect was to increase the water content of the small intestine.

\section{Profile-effect relationship investigation}

The profile-effect relationship between the nine common peaks of $S$. grosvenorii aqueous extracts and their laxative pharmacological activity including the incubation period of defecation, the number of defecations, the wet weight of defecation, and the dry weight of defecation are shown in Figs. 9, 10, 11 and 12, respectively. Figure 9 shows that the presence of seven common peaks (P1, P2, P3, P5, P6, P8, and P9) in the HPLC fingerprint of $S$. grosvenorii aqueous extracts was highly correlated with the incubation period of defecation in mice (correlation grade $>0.90$ ). The rank order for the contribution of each component to shortening the incubation period of defecation in mice was determined as $\mathrm{P} 5>\mathrm{P} 6, \mathrm{P} 9>\mathrm{P} 2>\mathrm{P} 1, \mathrm{P} 3, \mathrm{P} 8>\mathrm{P} 4>\mathrm{P} 7$. Figure 10 shows that the presence of eight common peaks (P1, P2, P3, P4, P5, P6, P8, and P9) in the HPLC fingerprint of $S$. grosvenorii aqueous extracts was highly correlated with the number of defecations in mice (correlation grade > 0.80 ). The rank order for the contribution of each component to increasing the number of defecations in mice was determined as $\mathrm{P} 5>\mathrm{P} 2>\mathrm{P} 6, \mathrm{P} 9>\mathrm{P} 1, \mathrm{P} 3, \mathrm{P} 8>\mathrm{P} 4>$ P7. Figure 11 shows that the presence of seven common peaks (P1, P2, P3, P5, P6, P8, and P9) in the HPLC fingerprint of $S$. grosvenorii aqueous extracts was highly correlated with the effect of the wet weight of defecation in mice (correlation grade $>0.80$ ). The rank order for the contribution of each component to increasing the wet weight of defecation in mice was determined as P5 > $\mathrm{P} 2>\mathrm{P} 6, \mathrm{P} 9>\mathrm{P} 3>\mathrm{P} 1, \mathrm{P} 8>\mathrm{P} 4>\mathrm{P} 7$. Figure 12 shows that the presence of seven common peaks (P1, P2, P3, P5, P6, P8, and P9) in the HPLC fingerprint of S. grosvenorii aqueous extracts was highly correlated with the dry weight of defecation in mice (correlation grade $>0.80$ ). The rank order for the contribution of each component to increasing the dry weight of defecation in mice was determined as $\mathrm{P} 5>\mathrm{P} 2>\mathrm{P} 9>\mathrm{P} 6>\mathrm{P} 1, \mathrm{P} 3, \mathrm{P} 8>\mathrm{P} 4>\mathrm{P} 7$. Of all the common peaks, P5 showed the highest correlation with the laxative pharmacological activity of the aqueous extracts. The only component whose identity was confirmed, P7 (mogroside V), which is part of the quality control index of $S$. grosvenorii in the Chinese Pharmacopoeia (2015 version), showed the worst correlation with the laxative pharmacological activity.

\section{Discussion}

Functional constipation is one of the most common chronic functional gastrointestinal diseases in clinical treatment. It is believed that the pathogenesis of constipation is complicated and various, which mainly relates to colonic dysfunction or intestinal absorption of excessive moisture [26]. Therefore, the treatment strategies for functional constipation should focus on the promotion of intestinal motility as well as the prevention of excessive absorption of water. The pharmacological effects of phytomedicine on promoting defecation mainly includes: (1) shortening the incubation period of defecation, increasing the number or weight of defecation, softening the stool and improving the fecal water content, (2) enhancing the contraction frequency or amplitude of the ileum, and promoting the propulsion of the small and large intestines, and (3) increasing the water content of the enteric cavity or myenteron. In the current study, S. grosvenorii from some places of origin were observed to significantly shorten the incubation period of defecation, and significantly increase the 


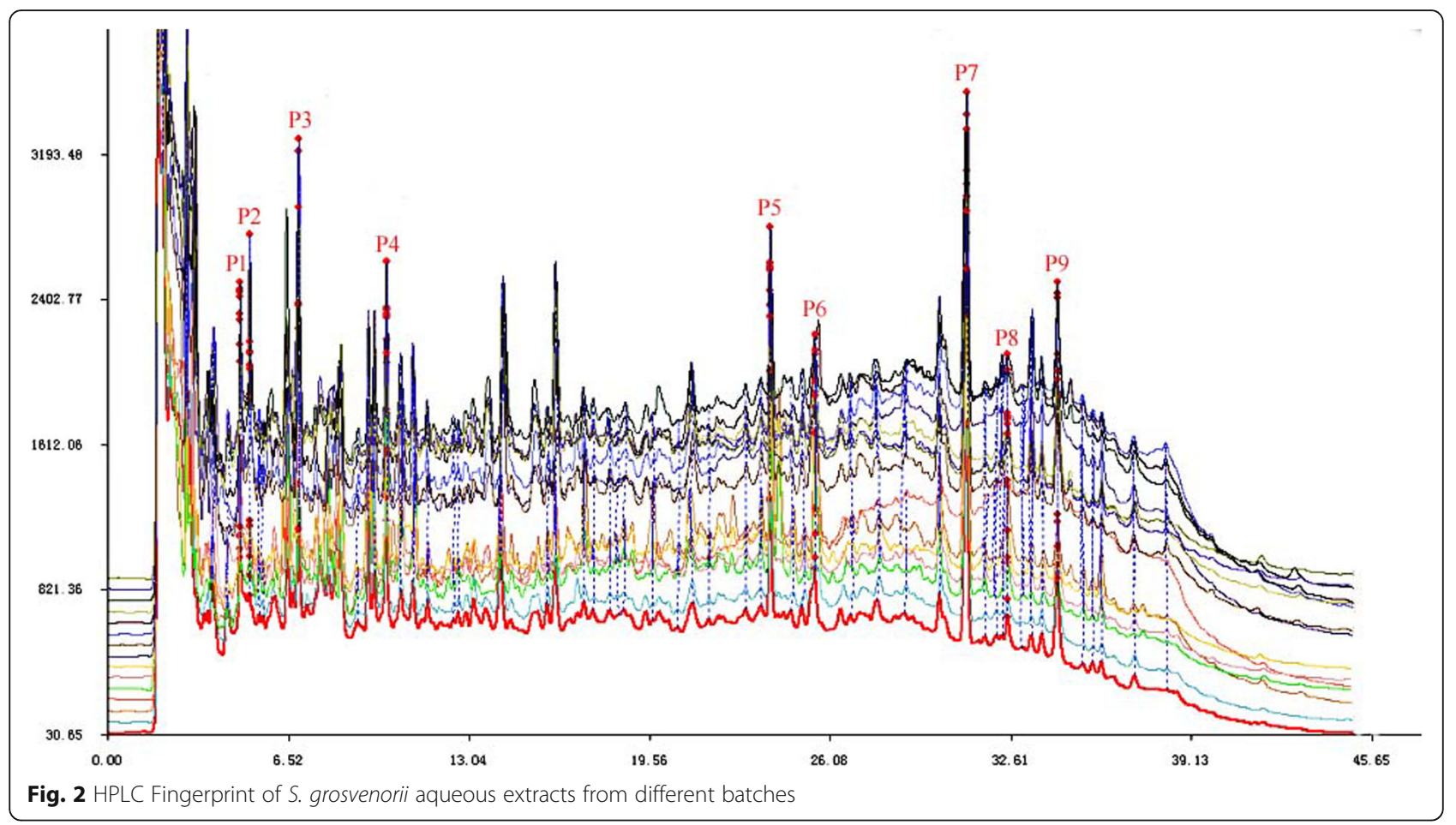

number of defecations, and the wet and dry weights of defecation in constipated mice. These encouraging results suggested the definite curative effects of S. grosvenorii in treating constipation, which were equivalent or better than that of mosapride. We concluded that a potential explanation for the laxative effect of the aqueous extracts of $S$. grosvenorii was related to their ability to increase the MTL level in the serum of mice, and thus promote gastric emptying and intestinal propulsion. Further, it increased the water content of the small

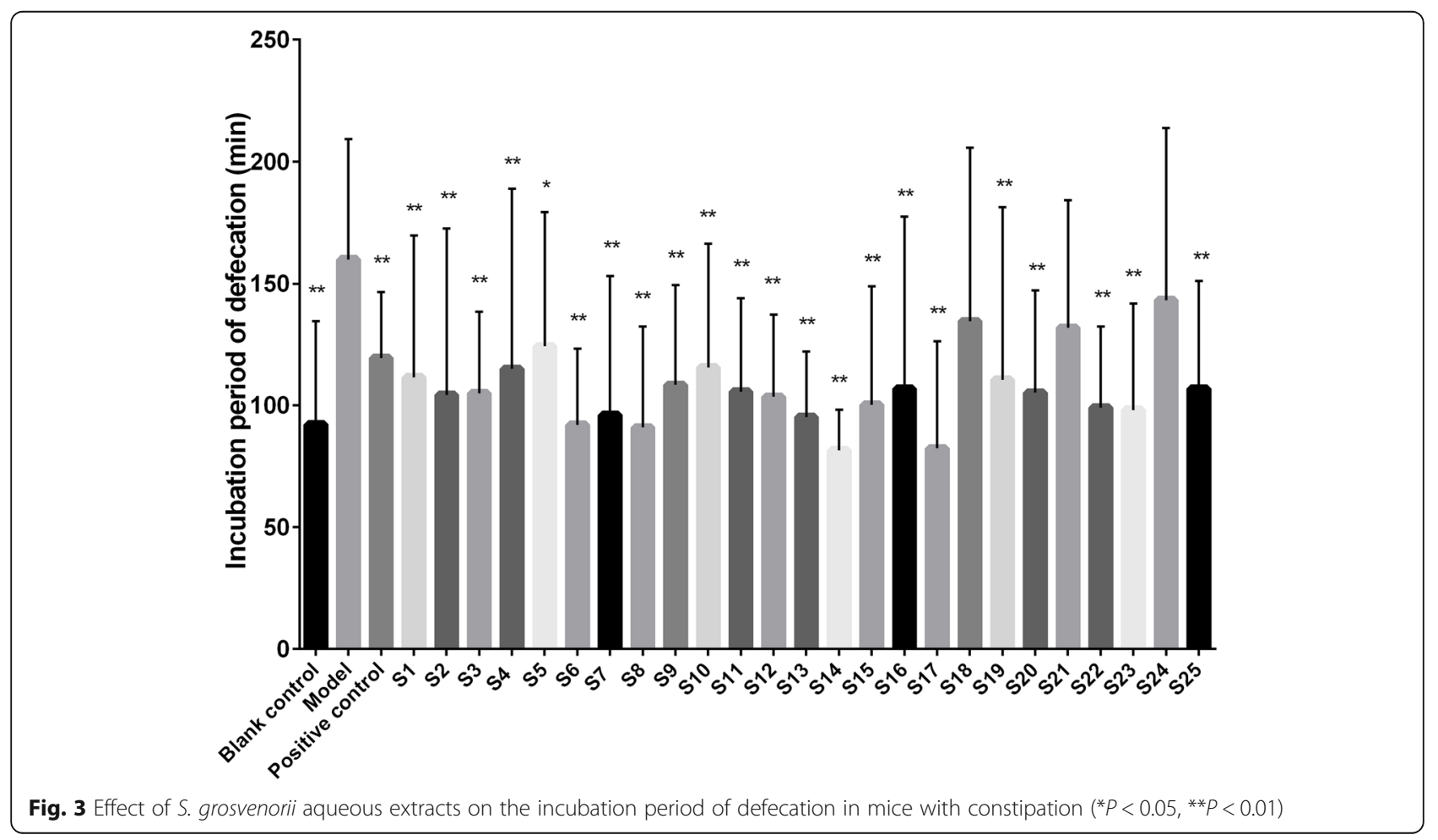




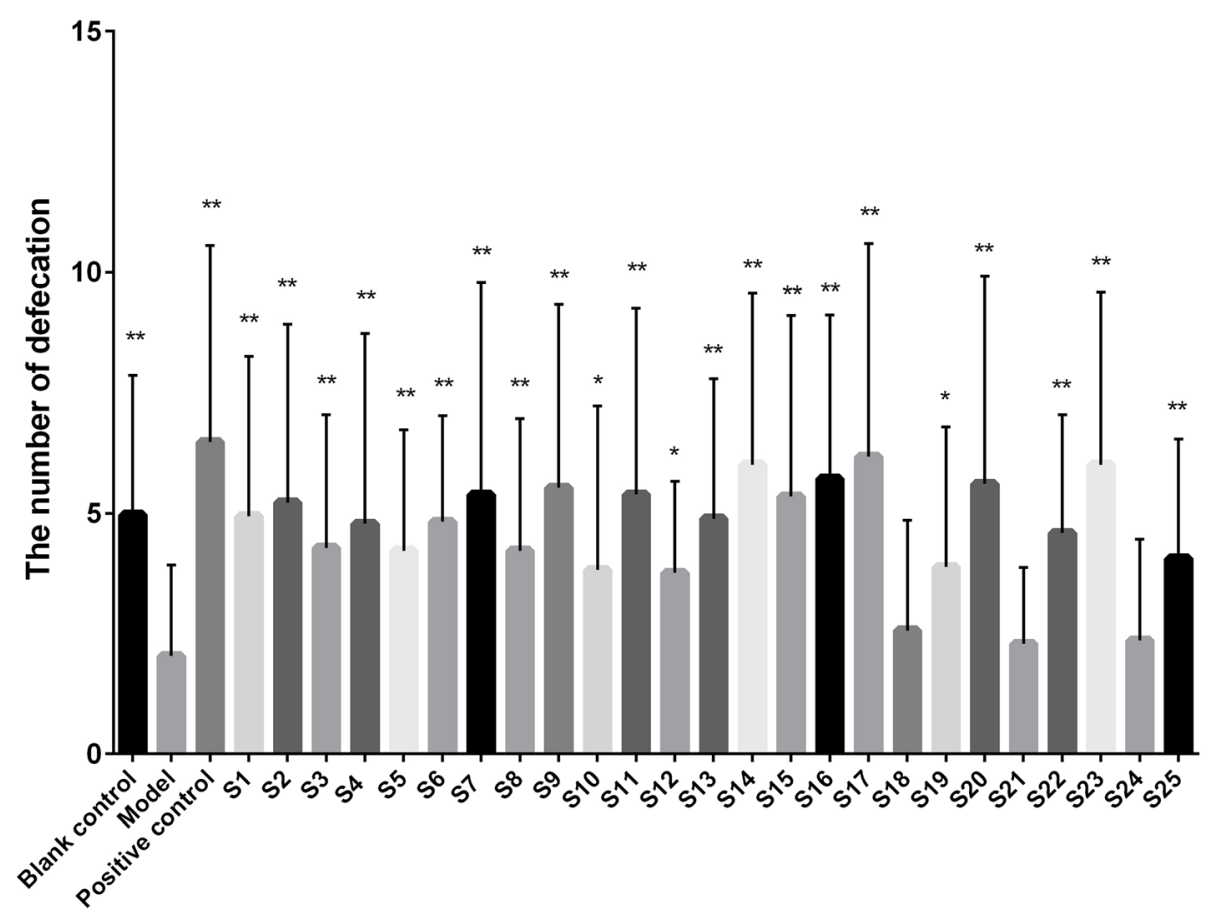

Fig. 4 Effect of S. grosvenorii aqueous extracts on the number of defecation in mice with constipation $\left({ }^{*} P<0.05,{ }^{*} P<0.01\right)$

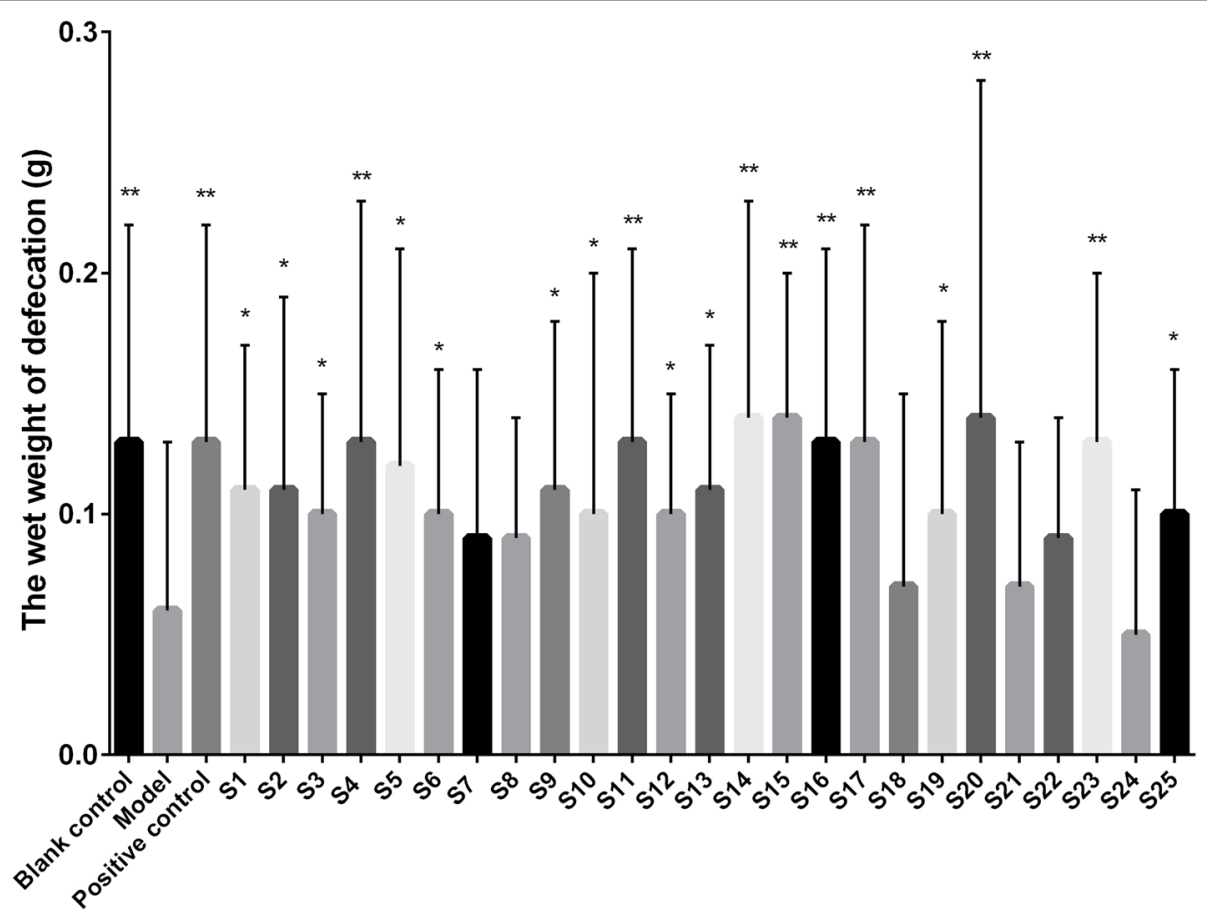

Fig. 5 Effect of $S$. grosvenorii aqueous extracts on the wet weight of defecation in mice with constipation $\left(* P<0.05,{ }^{* *} P<0.01\right)$ 


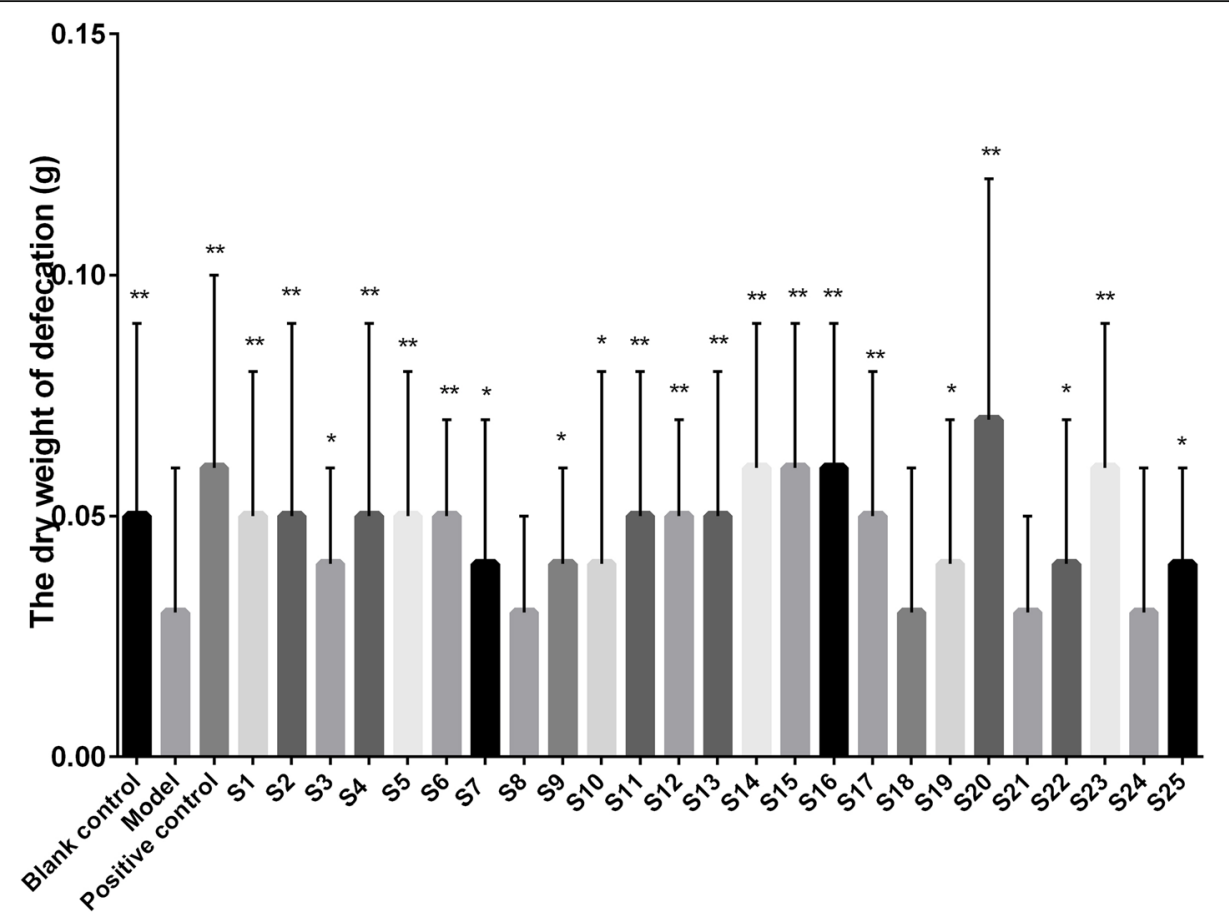

Fig. 6 Effect of S. grosvenorii aqueous extracts on the dry weight of defecation in mice with constipation $\left({ }^{*} P<0.05,{ }^{*} P<0.01\right)$

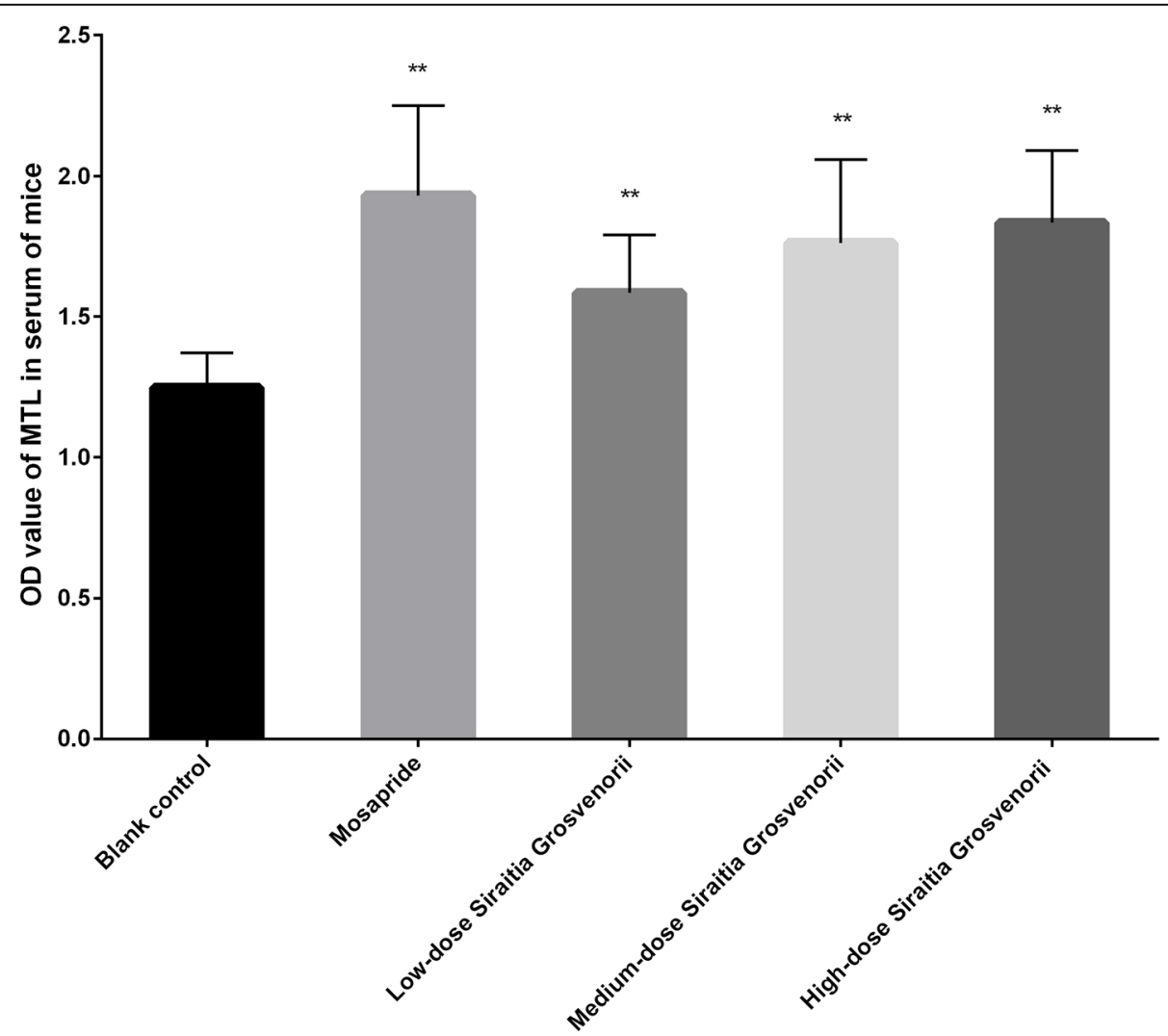

Fig. 7 Level of MTL in serum of mice treated with different dose of S. grosvenorii aqueous extracts $(* * P 0.01)$ 
intestine, which supported its effect on bowel relaxation. Nevertheless, the mechanism by which $S$. grosvenorii increased the content of serum MTL and the water content of the small intestine in mice requires more detailed investigations of the gene and protein expression levels.

Fingerprint similarity evaluation is a method used to estimate the similarity of chemical components of phytomedicine. Theoretically, herbals with high similarities in their chemical fingerprints should have similar chemical components and thus, similar efficacies. We found that the fingerprints of different batches of S. grosvenorii were highly similar although the main characteristic peaks were not completely consistent. As shown in Fig. 2 , nine common fingerprint peaks were identified from different batches. In the fingerprint, each characteristic peak represents a specific chemical component. The laxative effect of S. grosvenorii is the result of the synergistic action of its various chemical components. Hence, there must be some relationship between the fingerprint characteristics and the efficacy. However, the lack of a suitable method useful for identifying the components of $S$. grosvenorii that are efficacious is a critical gap in the quality control of S. grosvenorii. The use of simple bivariate correlation analysis and regression analysis are not applicable to explain the complex relationship between chemical composition and pharmacodynamics of S. grosvenorii. The gray correlation analysis provides an effective way to address this issue.

Gray relation analysis is a type of order relation analysis that has been successfully applied to decision- making, prediction, comprehensive evaluation, and accuracy testing of gray modeling. A large number of studies have confirmed that in research fields with less information and more interfering factors, gray relational analysis is one of the most promising mathematical approaches for order relation analysis. Currently, there are few studies on the application of gray correlation analysis in the quality evaluation of phytomedicine. In fact, this method is valuable because it uses known information to interpret unknown information. Therefore, it is suited for use in the quality evaluation and control of phytomedicine containing complex chemical components. As an exploratory analysis tool, gray correlation analysis provides a novel approach and shows great potential for standardization of $S$. grosvenorii for quality control purposes.

In the present work, we determined the contribution of the chemical components represented by the nine characteristic peaks of the fingerprint to the laxative effect of $S$. grosvenorii using gray correlation analysis. Since conventional research methods typically only allow the pharmacological effect of a single component or effective fractions of $S$. grosvenorii to be determined, the innovation of this work is in the establishment of a method useful to analyze the contribution of "effective component groups" to the laxative effect of $S$. grosvenorii. Considering that the effects of $S$. grosvenorii is not a simple linear superposition of its different components, in the future, we will use advanced analysis technology, powerful computer processing, and chemical

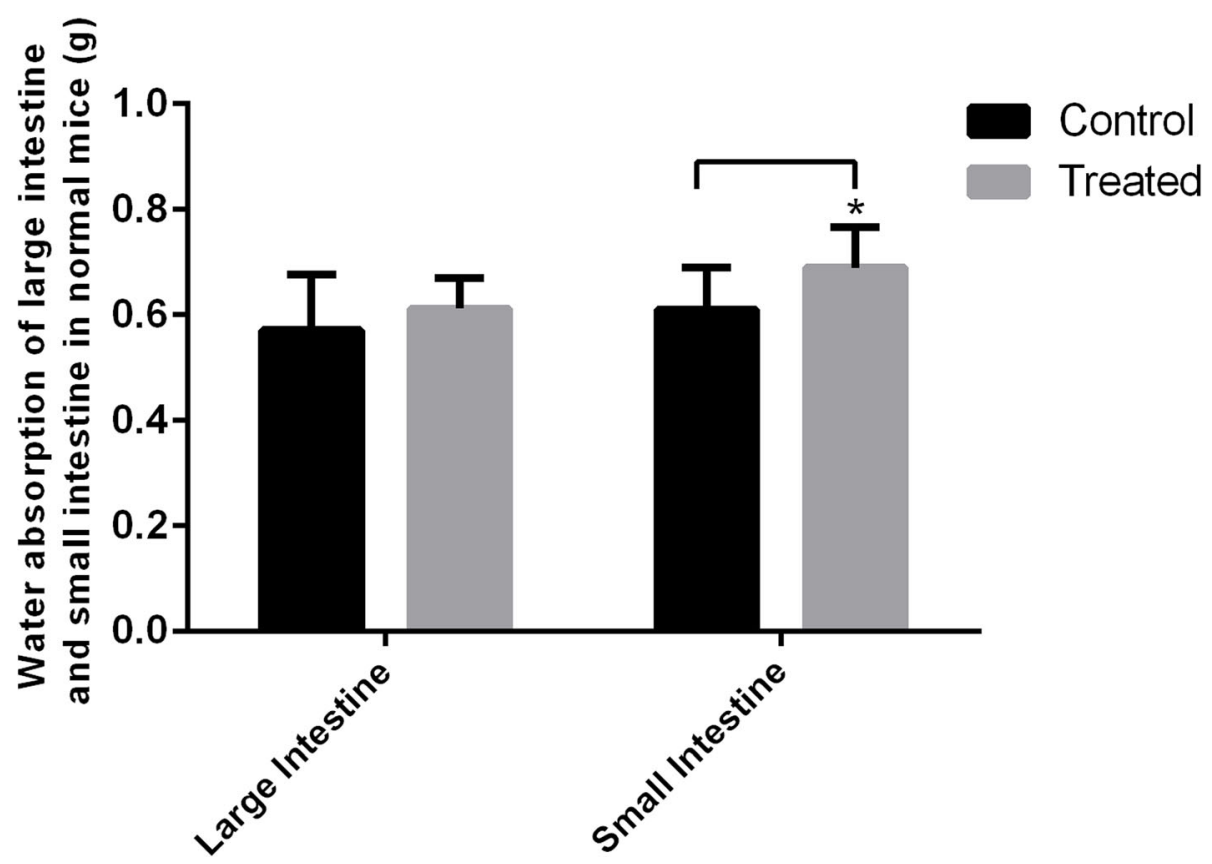

Fig. 8 Effect of S. grosvenorii aqueous extracts on water absorption of large and small intestines in normal mice $\left.{ }^{*} P<0.05\right)$ 


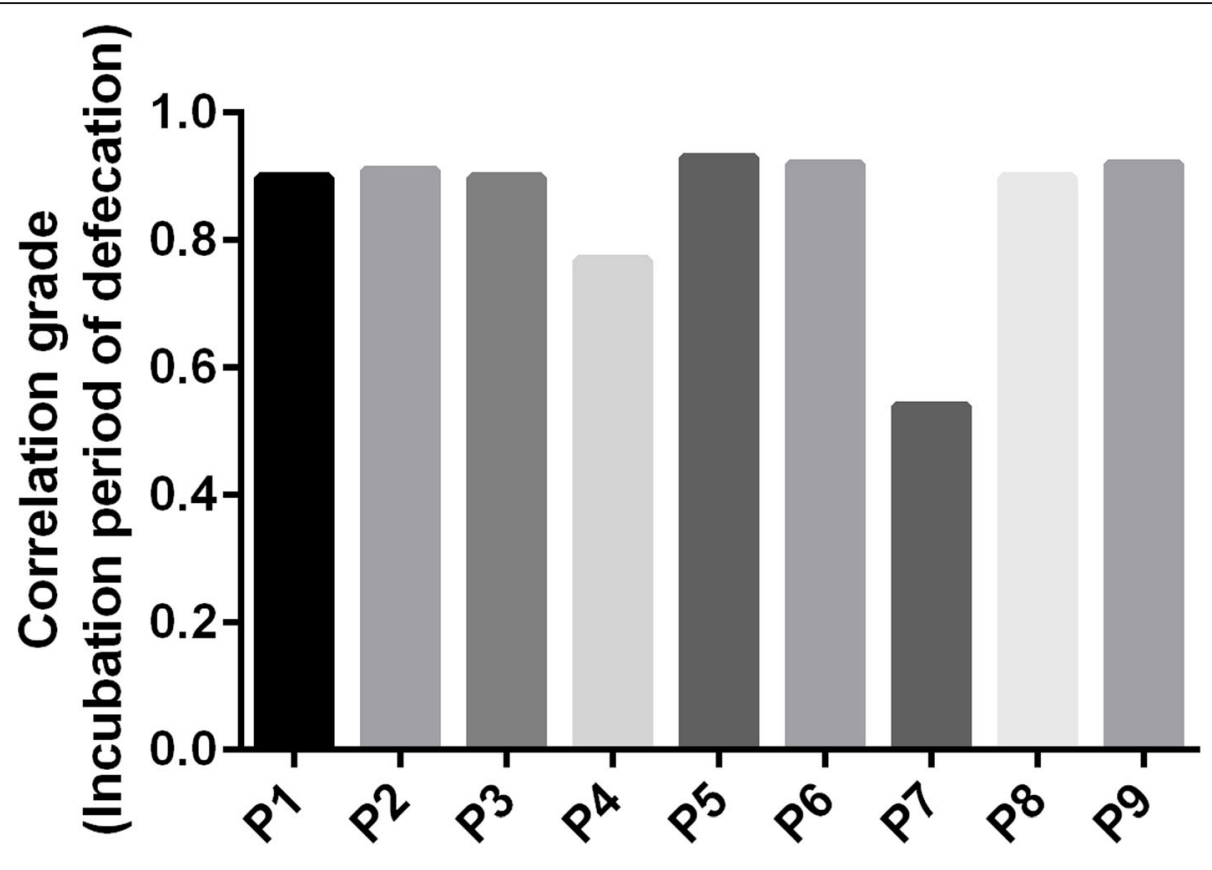

Common Peaks

Fig. 9 Correlation grade between the incubation period of defecation in mice with constipation and the common peaks in S. grosvenorii aqueous extracts

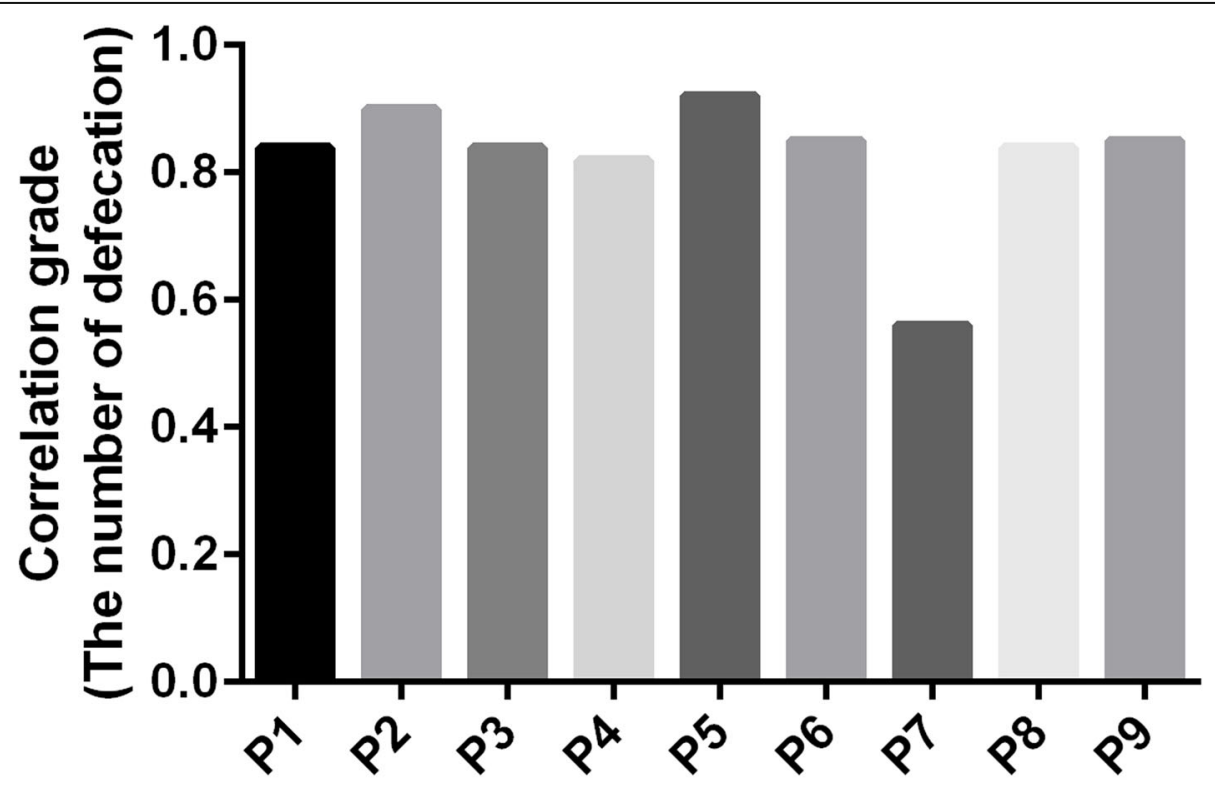

Common Peaks 


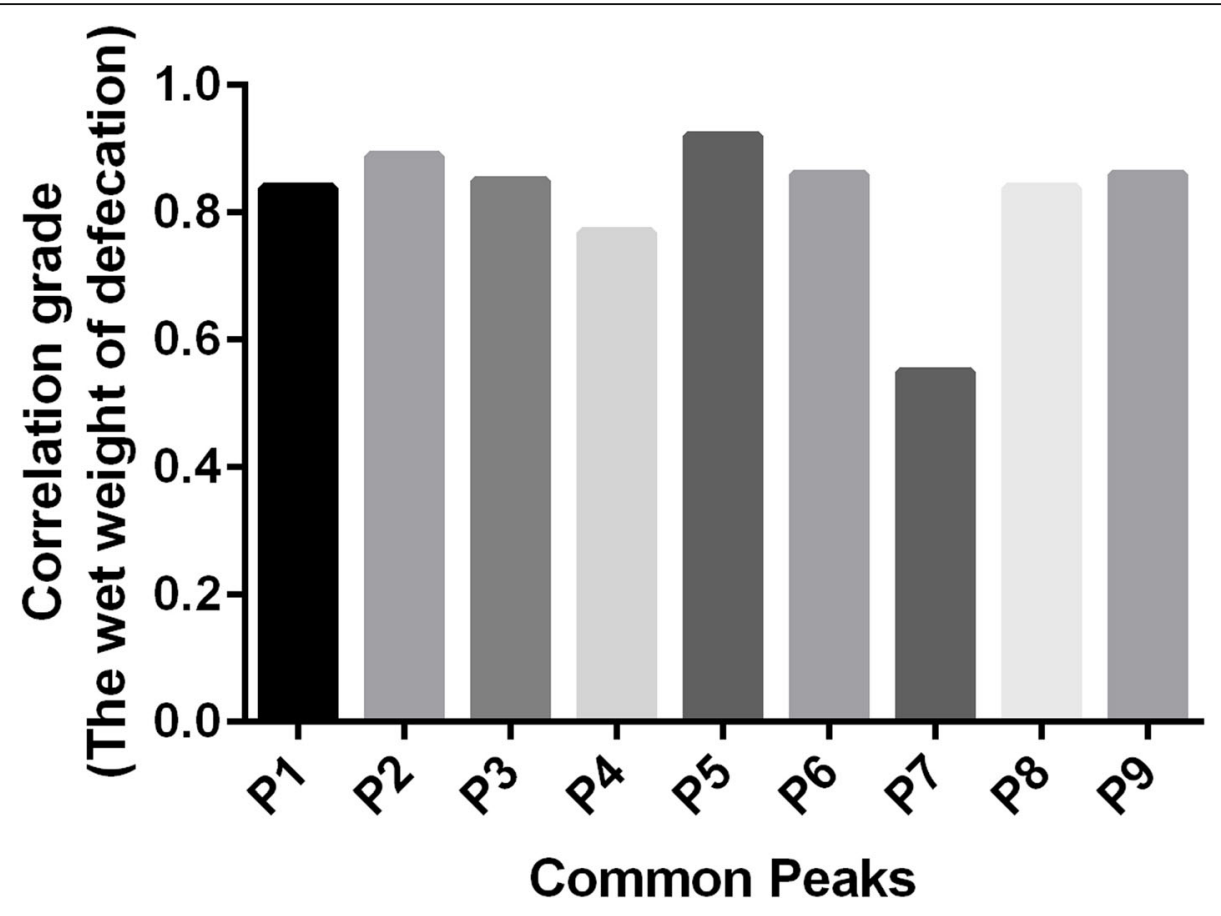

Fig. 11 Correlation grade between the wet weight of defecation in mice with constipation and the common peaks in S. grosvenorii aqueous extracts

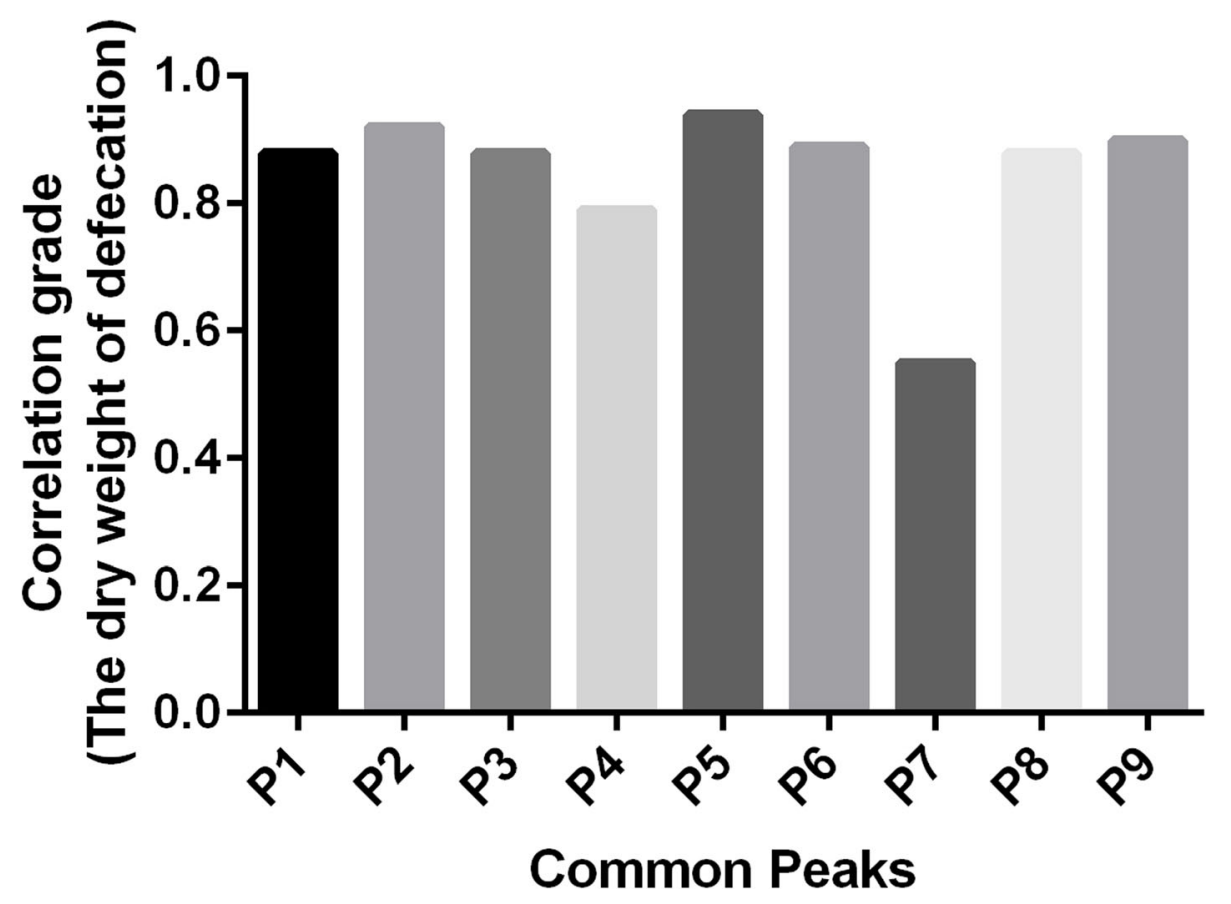

Fig. 12 Correlation grade between the dry weight of defecation in mice with constipation and the common peaks in S. grosvenorii aqueous extracts 
information technology to "decode" the fingerprints, and further clarify the relationship between the "profile" and the "effect". Additionally, except for Peak 7, we have not yet determined the specific chemical composition of the other eight common peaks. Especially, the structure and the phytochemical characteristic of Peak 5 are necessary to be elucidated, since it is the most relative one to the laxative effect. Through consulting related literatures [27, 28], we found that the main bioactive components of $S$. grosvenorii are a group of cucurbitane-type triterpene glycosides, normally named as mogrosides, mainly including mogroside III, mogroside IV, mogroside IVa, isomogroside V, mogroside VI, siamenoside I, mogroside V and 11-oxomogroside $\mathrm{V}$. These mogrosides have mogrol triterpenoid aglycone in common and differ in the sugars attached. Therefore, we speculate that the other eight peaks may belong to a certain kind of the mentioned mogrosides. Future research will focus on the identification of these peaks.

\section{Conclusion}

In the present study, nine common peaks were identified from the fingerprints of different batches of S. grosvenorii aqueous extracts by HPLC, making it a promising quality control method for S. grosvenorii. A pharmacological study of the laxative effect showed that S. grosvenorii aqueous extracts from 25 batches exhibited different therapeutic effects on mice with constipation because differences in their ecological environment, growth phase, breeding type and/or size fast influence the phytochemical profile resulting in different activities. Samples S4, S11, S14-S17, S20, and S23 obviously shortened the incubation period of defecation $(P<0.01)$, and significantly increased the number of defecations, the wet weight of defecation, and the dry weight of defecation in constipated mice $(\mathrm{P}<0.01)$. The mechanism may be related to the improvement of the MTL serum level and the water absorption of the small intestine in normal mice, thus promoting gastric emptying and small intestinal peristalsis. With the help of gray correlation analysis, the profile-effect relationship between the nine common peaks of $S$. grosvenorii aqueous extracts and their laxative pharmacological activity was confirmed and the rank order for the contribution of each component was determined. This study is of great significance to the establishment of quality control standards as well as the determination of the effective components of S. grosvenorii.

\section{Abbreviations}

MTL: Motilin; HRP: Horseradish peroxidase; TMB: Tetramethylbenzidine; OD: Optical density

\section{Supplementary Information}

The online version contains supplementary material available at https://doi. org/10.1186/s12906-021-03388-X.

\section{Additional file 1.}

\section{Acknowledgements}

Not applicable.

\section{Authors' contributions}

WD and XJ performed the experiments. QW designed and supervised the study. JZ and TH were involved with data analysis and wrote the manuscript. All authors read and approved the final manuscript.

\section{Funding}

Not applicable.

\section{Availability of data and materials}

The datasets from the present study are available from the corresponding author on reasonable request.

\section{Declarations}

Ethics approval and consent to participate

All experiments were performed according to the guidelines for the care and use of animals established by Guangxi University of Chinese Medicine. All animal studies were in line with the guidelines of the Animal Ethics Committee of Guangxi University of Chinese Medicine.

\section{Consent for publication}

Not applicable.

\section{Competing interests}

The authors declare that they have no competing interests.

\section{Author details}

'Department of Pharmacy, The Affiliated Hospital of Jiaxing University, Department of Pharmacy, The First Hospital of Jiaxing, Jiaxing 314000, Zhejiang Province, PR China. ${ }^{2} \mathrm{NHC}$ Key Laboratory of Reproduction Regulation, Shanghai Engineering Research Center of Reproductive Health Drug and Devices, Shanghai Institute of Planned Parenthood Research, Shanghai 200032, PR China. ${ }^{3}$ Department of Pharmacy, Guangxi University of Chinese Medicine, Nanning 530001, Guangxi Province, PR China.

Received: 10 December 2020 Accepted: 10 August 2021

Published online: 20 September 2021

\section{References}

1. Hamza AA, Ahmed MM, Elwey HM, Amin A. Melissa officinalis protects against doxorubicin-induced cardiotoxicity in rats and potentiates its anticancer activity on MCF-7 cells. PLoS One. 2016;11(11):e0167049. https:// doi.org/10.1371/journal.pone.0167049.

2. Hrout AA, Chaiboonchoe A, Khraiwesh B, Murali C, Baig B, El-Awady R, et al. Safranal induces DNA double-strand breakage and ER-stress-mediated cell death in hepatocellular carcinoma cells. Sci Rep. 2018;8:16951.

3. Ashktorab H, Soleimani A, Singh G, Amin A, Tabtabaei S, Latella G, et al. Saffron: the golden spice with therapeutic properties on digestive diseases. Nutrients. 2019;11(5):943. https://doi.org/10.3390/nu11050943.

4. Murali C, Mudgil P, Gan CY, Tarazi H, El-Awady R, Abdalla Y, et al. Camel whey protein hydrolysates induced G2/M cellcycle arrest in human colorectal carcinoma. Sci Rep. 2021;11:7062.

5. Amin A, Mahmoud-Ghoneim D. Texture analysis of liver fibrosis microscopic images: a study on the effect of biomarkers. Acta Biochim Biophys Sin. 2011;43(3):193-203. https://doi.org/10.1093/abbs/gmq129.

6. Pharmacopoeia Committee of People's Republic of China. Pharmacopoeia of the People's Republic of China, voll. Beijing: China Medical Science and Technology Press; 2015.

7. Li C. Siraitia grosvenorii Swingle, Dietary Chinese Herbs. Switzerland: Springer Link; 2015. p. 431-8.

8. Li C, Lin LM, Sui F, Wang ZM, Huo HR, Dai L, et al. Chemistry and pharmacology of Siraitia grosvenorii: A review. Chin J Nat Med. 2014;12(2): 89-102. https://doi.org/10.1016/S1875-5364(14)60015-7. 
9. Nong $\mathrm{YQ}$, Jiang L. An overview of research on the extraction and pharmacologic action of mogrosides. Guangxi J Tradit Chin Med. 2008;31(1): 6-8.

10. Si JY, Chen DH, Shen LG. Isolation and structure determination of flavonol glycosides from the + fruits of Siraitia grosvenorii. Acta Pharm Sin. 1994; 29(2):158-60.

11. Chen QB, Yang JX, Cheng ZQ, Yi XH. The determination of total falvonoids in Momordica grosvenorii leaf by RP-HPLC. Guangxi J Tradit Chin Med. 2005;24(5):133-5.

12. Wang Q, Li AY, Li XP, Zhang J, Liang DB, Qiu FL, et al. Pharmacological effects of Siraitia grosvenorii fruit. Chin J Chin Mater Med. 1999;24(7):425-8.

13. Wang T, Huang ZJ, Jiang YM. Studies on the pharmacological profile of mogrosides. Chin Tradit Herb Drugs. 1999;30(12):914-6.

14. Liu T, Wang XH, Chun L, Zhang Y, Wu GL, Li DC, et al. Study on the antitussive, expectorant and antispasmodic effects of Saponin $\vee$ from Momordica grosvenorii. Chin Pharma J. 2007:42(20):1534-6.

15. Wang Q, Wang K, Dai SM, Yang YQ, Chen YJ, Mo ZH. Regulation on the immunological effect of mogrosides in the mice. J Chin Med Mat. 2001; 24(11):811-2.

16. Li J, Huang Y, Liao RQ, He XC, Su XJ, Zhong ZX, et al. Effect of Siraitia grosvenorii polysaccharide on immunity of mice. Chin Pharmacol Bull. 2008; 24(9):1237-40

17. Matsumoto S, Jin M, Dewa Y, Nishimura J, Moto M, Murata Y, et al. Suppressive effect of Siraitia grosvenorii extract on dicyclanilpromoted hepatocellular proliferative lesions in male mice. J Toxicol Sci. 2009;34(1): 109-18. https://doi.org/10.2131/jts.34.109.

18. Wang Q, Ming X, Gang YJ. Experimental study of protective effect of Mog on chronic liver injury of rats. Guangxi J Tradit Chin Med. 2007;30(5):54-6.

19. Qi XY, Chen WJ, Zhang LQ, Xie BJ. Mogrosides extract from Siraitia grosvenorii scavenges free radicals in vitro and lowers oxidative stress, serum glucose, and lipid levels in alloxan-induced diabetic mice. Nutr Res. 2008;28(4):278-84. https://doi.org/10.1016/j.nutres.2008.02.008.

20. Suzuki YA, Murata YJ, Inui H, Sugiura M, Nakano Y. Triterpene glycosides of Siraitia grosvenorii inhibit rat intestinal maltase and suppress the rise in plasma glucose level after single oral administration of maltose in rats. $J$ Agric Food Chem. 2005;53(8):2941-6. https://doi.org/10.1021/jf0478105.

21. Suzuki YA, Tomoda M, Murata Y, Inui H, Sugiura M, Nakano Y. Antidiabetic effect of long-term supplementation with Siraitia grosvenorii on the spontaneously diabetic Goto-Kakizaki rat. Br J Nutr. 2007:97(4):770-5. https:// doi.org/10.1017/S0007114507381300.

22. Lin GP, Jiang T, Hu XB, Qiao XH, Tuo QH. Effect of Siraitia grosvenorii polysaccharide on glucose and lipid of diabetic rabbits induced by feeding high fat/high sucrose chow. Exp Diab Res. 2007;2007:1-4. https://doi.org/1 $0.1155 / 2007 / 67435$.

23. Pan MH, Yang JR, Tsai ML, Sang SM, Ho CT. Anti-inflammatory effect of Momordica grosvenorii Swingle extract through suppressed LPS-induced upregulation of INOS and COX-2 in murine macrophages. J Funct Foods. 2009:1 (2):145-52. https://doi.org/10.1016/j.jff.2009.01.003.

24. Takasaki M, Konoshima T, Murata Y, Sugiura M, Nishino H, Tokuda H, et al. Anticarcinogenic activity of natural sweeteners, cucurbitane glycosides from Momordica grosvenorii. Cancer Lett. 2003;198(1):37-42. https://doi.org/10.1 016/S0304-3835(03)00285-4.

25. Yao JW, Tang H, Shen WH, Li YX, Ding XS, Li XJ. The observation on impacts of the different dosage of Luo Han Guo on physiological function in mice by training of increasing intensity. Liaoning Sports Sci Technol. 2007;29(3): 24-6.

26. Zia-UI-Haq M, Dewanjee S, Riaz M. Past, Present and Future of Carotenoids Research. In: Carotenoids: Structure and Function in the Human Body. Cham: Springer; 2021. https://doi.org/10.1007/978-3-030-46459-2 28.

27. Li DP, Ikeda T, Nohara T, Liu JL, Wen YX, Sakamaoto T, et al. Cucurbitane glycosides from unripe fruits of Lo Han Kuo (Siraitia grosvenori). Chem Pharm Bull. 2007;55(7):1082-6. https://doi.org/10.1248/cpb.55.1082.

28. Zhang HY, Yang HH, Zhang $M$, Wang YR, Wang JG, Yao LF, et al. Identification of flavonol and triterpene glycosides in Luo-Han-Guo extract using ultra-high performance liquid chromatography/quadrupole time-offlight mass spectrometry. J Food Compos Anal. 2012;25(2):142-8. https://doi. org/10.1016/j.jfca.2011.09.004

\section{Publisher's Note}

Springer Nature remains neutral with regard to jurisdictional claims in published maps and institutional affiliations.

Ready to submit your research? Choose BMC and benefit from:

- fast, convenient online submission

- thorough peer review by experienced researchers in your field

- rapid publication on acceptance

- support for research data, including large and complex data types

- gold Open Access which fosters wider collaboration and increased citations

- maximum visibility for your research: over $100 \mathrm{M}$ website views per year

At BMC, research is always in progress.

Learn more biomedcentral.com/submissions 